\title{
Recurrence of SARS-CoV-2 RNA in upper respiratory tract samples from recovered patient with coronavirus disease 2019 (COVID-19)
}

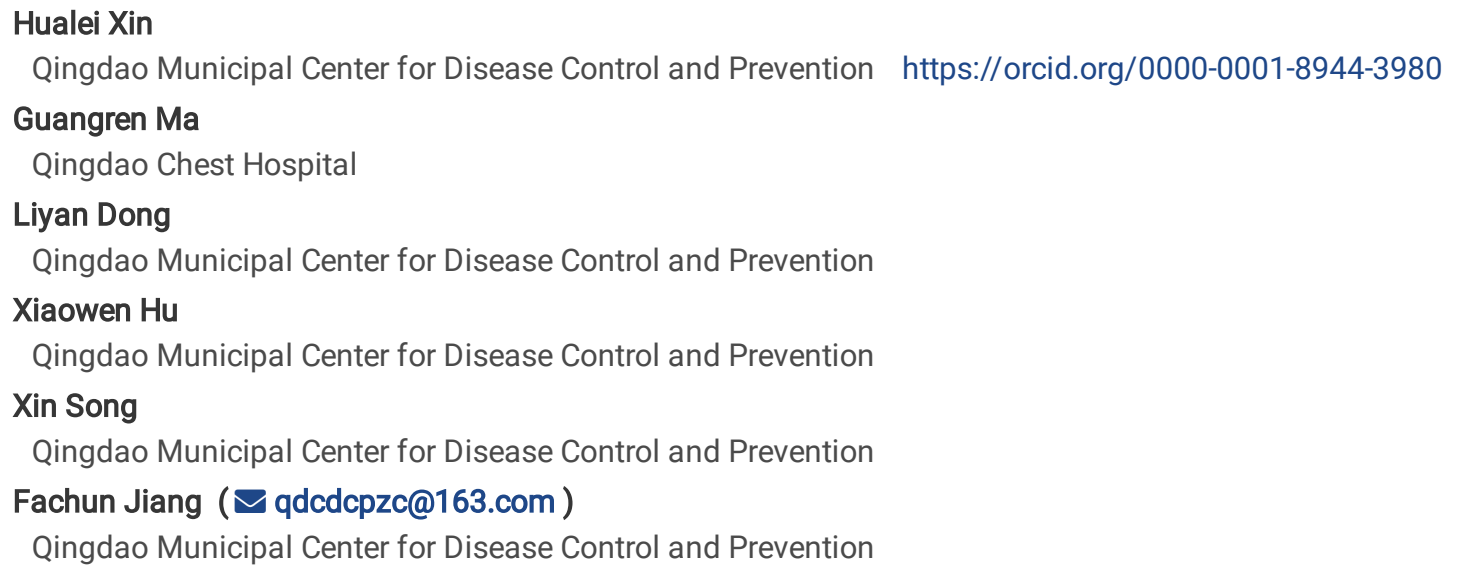

\section{Case Report}

Keywords: COVID-19, SARS-CoV-2, Recurrent, infectiousness, discharged

Posted Date: June 4th, 2020

DOI: https://doi.org/10.21203/rs.3.rs-32813/v1

License: (c) (i) This work is licensed under a Creative Commons Attribution 4.0 International License. Read Full License 


\section{Abstract}

Background

Case isolation and contacts tracing are the most widely used strategies to control the outbreak of COVID-19. However, little attention has been paid to the infectiousness of recovered patients with COVID-19.

Case presentation

In this study, we reported a confirmed case of COVID-19 whose nasopharyngeal swab test of SARS-CoV-2 RNA turned positive 28 days after hospital discharged.

Conclusions

SARS-CoV-2 RNA can be detected in respiratory tract sample 28 days after hospital discharged. Further studies about consecutive detection of SARS-CoV-2 combined with viral isolation among COVID-19 cases should be designed to determine the accurate contagious period.

\section{Background}

The coronavirus disease 2019 (COVID-19) caused by severe acute respiratory syndrome coronavirus 2 (SARS-CoV-2) has been responsible for over 4 million confirmed cases in more than 200 countries as of 14 May, 2020, and the number is still increasing [1]. On 30 January 2020, this outbreak was declared a Public Health Emergency of International Concern and on March 11 [2], the WHO Director-General made the assessment that COVID-19 can be characterized as a pandemic [2]. Case isolation and contact tracing are the most effective measures to against the spread of this outbreak. However, little attention has been paid to the follow-up of recovered patients. Limited studies have revealed that SARS-CoV-2 nuclear acid can be detected from rectal swabs or respiratory samples in patients recovered from COVID-19 [3-6]. In this study, we followed up one patient who had positive respiratory RT-PCR results 28 days after recovery from COVID-19 disease and tried to discuss the possible explanation and response measures.

\section{Methods}

Since 20 March 2020, all abroad people came to Qingdao Municipal should be quarantined in single hotel room for 14 days and two times of RT-PCR test for respiratory sample should be implemented during quarantine. Persons with positive results would be transported into designated hospital for isolation and treatment. The COVID-19 confirmed case was defined as a person presented with fever or other respiratory symptoms and had a positive result of SARS-CoV-2 detection in respiratory specimens by real-time RT-PCR methods [7]. The COVID-19 asymptomatic case was defined as a person presented with positive SARS-CoV-2 detection in respiratory or other specimens by real-time RT-PCR methods without clinical symptoms [7]. All following criteria should be met for hospital discharge: 1) normal temperature lasting longer than 3 days, 2) resolved respiratory symptoms, 3) substantially improved acute exudative lesions on chest computed tomography (CT) images, and 4) two consecutively negative RT-PCR test results separated by at least 1 day [8]. The illness severity of COVID19 was classified as mild, normal, severe and critical according to the Chinese diagnosis and treatment guidance of COVID-19 (version 7.0) [8].

The RT-PCR tests were performed on nasopharyngeal swabs and stool samples based on the universal methods [9]. The RT-PCR test kits were recommended by the Chinese Center for Disease Control and Prevention. Same technician and brand of test kit was used for both positive and negative samples. The cycle threshold values less than 40 is interpreted as positive for SARS-CoV-2 RNA.

Demographic and epidemiological information were collected from epidemiological reports and National Notifiable Infectious Disease Reporting Information Symtem (NNIDIRS). Clinical symptoms, treatment, laboratory test (routine blood examination, biochemical test, antibody test for COVID-19 and other respiratory pathogens) and radiological features were extracted from electronic medical records. Date of RT-PCR test results were collected from laboratory registration form in the Qingdao Municipal Center for Disease Control and Prevention.

\section{Case Presentation}

On 24 March 2020, local center for disease control and prevention (cdc) performed routine RT-PCR test for COVID-19 nucleic acid in nasopharyngeal swab samples among quarantined abroad people, and a positive result was identified. The patient is a 19 years-old Chinese female with underlying disease of myocarditis. She has become an oversea student in England since September 2019. On March 19, 2020 she came back to China by taking plane and self-reported a man sitting next to her on the plane experienced serious cough during the journey. However, we can't determine whether this man was COVID-19 case because he got off plane in Singapore. On March 21 she arrived

Page 2/5 
in China and quarantined in single hotel room. The patient was initially asymptomatic with normal CT imaging and routine blood examination before March 26. On March 27 the patient developed fever $\left(37.5^{\circ} \mathrm{C}\right)$, slight cough and running nose and CT imaging showed scattered mottling opacity in the left lung. On April 1, the patient was diagnosed as COVID-19 confirmed case and the severity of disease was normal.

On admission, antiviral treatment including interferon a 5 million units of taken aerosol inhalation every 12 hours and ribavirin 300 mg of taken orally every 8 hours, was provided for the patient, and the treatment procedure lasted for 12 days. Other adjunctive therapy such as oxygen supplemented and traditional Chinese medicine therapy were also provided. The patient's body temperature persistently normal since hospitalization, and respiratory symptoms (cough) were significantly relieved and completely disappeared on April 13, and CT imaging abnormalities had resolved since April 3. Results from routine blood examination and biochemical tests were normal throughout disease course and normal vital signs with oxygen saturation of $98 \%$ while the patient was breathing ambient air. IgM test for influenza $A$ and $B$, parainfluenza, respiratory syncytial virus, adenovirus, coxsackievirus, Mycoplasma pneumoniae, and Chlamydia pneumoniae was negative. IgM and IgG colloidal gold test for COVID-19 was negative on April 2, while the IgG turned positive on April 7. Four times of RT-PCR test were performed during hospitalization including 2 positive results (March 29 and April 9) and 3 negative results (April 1, April 11 and April 12), she discharged on April 13. The time from symptom onset to negative result of nucleic acid test was 16 days and the length of hospital stay was 21 days.

After hospital discharge, the patient continued to be quarantined at designated hospital for 14 days (April 13- April 26). She experienced asymptomatic and normal CT imaging throughout the quarantined period, and the routine blood examination and biochemical tests were also normal. RT-PCR tests for nasopharyngeal swab and stool were performed in 10th and 14th days of quarantine and all were negative. After discontinuation of quarantine at hospital, the patient was asked to continue the quarantine protocol at home for 14 days (April 27 May 11). Positive RT-PCR test results were identified in respiratory sample on May 11 and May 12 while the results in stool was negative. The patient continued to be asymptomatic by clinician examination and chest CT findings showed no change from previous images. However, routine blood examination revealed abnormal white blood cell count $\left(10.85 \times 10^{9}\right.$, reference range: $\left.4-10 \times 10^{9}\right)$. Two following RT-PCR tests were performed on May 13 and May 15, and negative result were identified in both respiratory and stool samples. Two household contacts (the patient's family member) were traced and sampled and none of them was infected.

\section{Discussion}

We speculate on the reasons why the SARS-CoV-2 nuclear acid was detected in the hospital discharged COVID-19 case. No study has accurately established the contagious period of COVID-19 and SARS-CoV-2 RNA from respiratory tract specimens may be persistent or recurrently positive during the course of this disease. The angiotensin-converting enzyme-2 (ACE-2), which is the cell entry receptor of SARSCoV-2, was highly expressed in lower respiratory tract rather than in the upper respiratory tract [10]. The result of the SARS-CoV-2 RNA test likely depends on viral load of the specimen [5]. Therefore, false negatives for nasopharyngeal swab tests may occur, affected by the experience of the operator, site from which the sample was taken and the actual quantity of virus.

Although SARS-CoV-2 RNA were found among convalescent COVID-19 patients, however, all nuclear acid test of COVID-19 was performed by RT-PCR method. An important limitation of PCR testing, however, is the inability to differentiate between actual viral replication and the detection of non-viable viral material [6]. This has been a key challenge when assessing the infectiveness of recovering patients and determining the clinical course of infectious diseases. In addition, until now, no research revealed that the alive viral was isolated from respiratory tract samples among recovered patients. Recently, a screening survey conducted in Wuhan Municipal (the most endemic area in China), Hubei Province found 659 asymptomatic carriers and 559 of them were recovered patients. Fifty-five respiratory tract samples from

the asymptomatic carriers were selected for viral isolation and all were negative. Besides, until now, no confirmed or asymptomatic COVID-19 cases were identified among contacts of recovered COVID-19 patient with positive result of SARS-CoV-2 RNA [3-5, 11, 12]. In terms of disease containment, it is better that patients should be kept in isolation as long as they show signs of viral RNA shedding. However, based on the impact on health care systems, virology laboratories, and patients would be enormous if kept isolation for a long time, it may be necessary to accept some uncertainties and to rely on clinical improvement from COVID-19 and the current discharge criteria to inform strategies for ending isolation.

This study had two limitations. Firstly, viral isolation wasn't done, therefore we couldn't determine the infectiousness of this case. Secondly, limited times of RT-PCR test was done during the quarantine period.

\section{Conclusions}


A confirmed patient with COVID-19 who met all criteria for hospital discharge in Qingdao Municipal had positive RT-PCR test results for respiratory samples 28 days later after hospital discharge. This finding, with the addition of other studies, suggest that at least a proportion of recovered patients still may be tested positive for SARS-CoV-2. Further study of consecutively RT-PCR test combine with viral isolation in lower respiratory tract samples among COVID-19 cases should be designed to determine the accurate contagious period of COVID-19.

\section{Declarations}

\section{Acknowledgments}

We gratefully thank the staff members of the county-level Centers for Disease Control and Prevention of Qingdao for their assistance in the data collection, validation and cleanup.

\section{Ethical approval and Consent to participate}

The study was approved by the Ethics Committee of Qingdao Municipal Center for Disease Control and Prevention and all data patients used in this study were anonymized so that individual patients could not be identified.

\section{Consent for publication}

Not applicable.

\section{Availability of data and material}

All data generated or analysed during this study are included in this published article and its supplementary information files.

\section{Competing interests}

The authors declare that they have no competing interests.

\section{Funding}

Not applicable.

\section{Authors' contributions}

All authors have contributed significantly to the work and have seen and approved this version of the manuscript.

\section{Authors' information}

All authors' information are listed on the title page.

\section{References}

1. World Health Organization. Coronavirus disease (COVID-19) Pandemic. 2020 [cited 2020 May 14]. https://www.who.int/emergencies/diseases/novel-coronavirus-2019.

2. World Health Organization. Rolling updates on coronavirus disease (COVID-19). [cited 2020 May 14]. https://www.who.int/emergencies/diseases/novel-coronavirus-2019/events-as-they-happen.

3. Zhang B, Liu S, Dong Y, Zhang L, Zhong Q, Zou Y, et al. Positive rectal swabs in young patients recovered from coronavirus disease 2019 (COVID-19). J Infect. 2020.

4. Lan L, Xu D, Ye G, Xia C, Wang S, Li Y, et al. Positive RT-PCR Test Results in Patients Recovered From COVID-19. JAMA. 2020.

5. Chen D, Xu W, Lei Z, Huang Z, Liu J, Gao Z, et al. Recurrence of positive SARS-CoV-2 RNA in COVID-19: A case report. Int J Infect Dis. 2020;93:297-9.

6. Barth RE, De Regt MJA. Persistence of viral RNA in stool samples from patients recovering from covid-19. Bmj. 2020;369:m1724.

7. National Health Commission of the People's Republic of China. Chinese management guideline for COVID-19 (version 6.0). In Chinese. Accessed May 14. 2020. http://www.nhc.gov.cn/yzygj/s7653p/202002/8334a8326dd94d329df351d7da8aefc2/files/b218cfeb1bc54639af227f922bf6b817.pdf.

8. National Health Commission of the People's Republic of China. Diagnosis and treatment of 2019-nCoV pneumonia in China (version 7.0). In Chinese. Accessed May 14. 2020. http://www.nhc.gov.cn/yzygj/s7653p/202002/d4b895337e19445f8d728fcaf1e3e13a.shtml. 
9. Wang D, Hu B, Hu C, Zhu F, Liu X, Zhang J, et al. Clinical Characteristics of 138 Hospitalized Patients With 2019 Novel CoronavirusInfected Pneumonia in Wuhan, China. Jama. 2020.

10. Lu R, Zhao X, Li J, Niu P, Yang B, Wu H, et al. Genomic characterisation and epidemiology of 2019 novel coronavirus: implications for virus origins and receptor binding. Lancet. 2020;395:565-74.

11. Zheng KI, Wang X-B, Jin X-H, Liu W-Y, Gao F, Chen Y-P, et al. A Case Series of Recurrent Viral RNA Positivity in Recovered COVID-19 Chinese Patients. Journal of General Internal Medicine. 2020.

12. Jiang M, Li Y, Han M, Wang Z, Zhang Y, Du X. Recurrent PCR positivity after hospital discharge of people with coronavirus disease 2019 (COVID-19). J Infect. 2020.

\section{Figures}

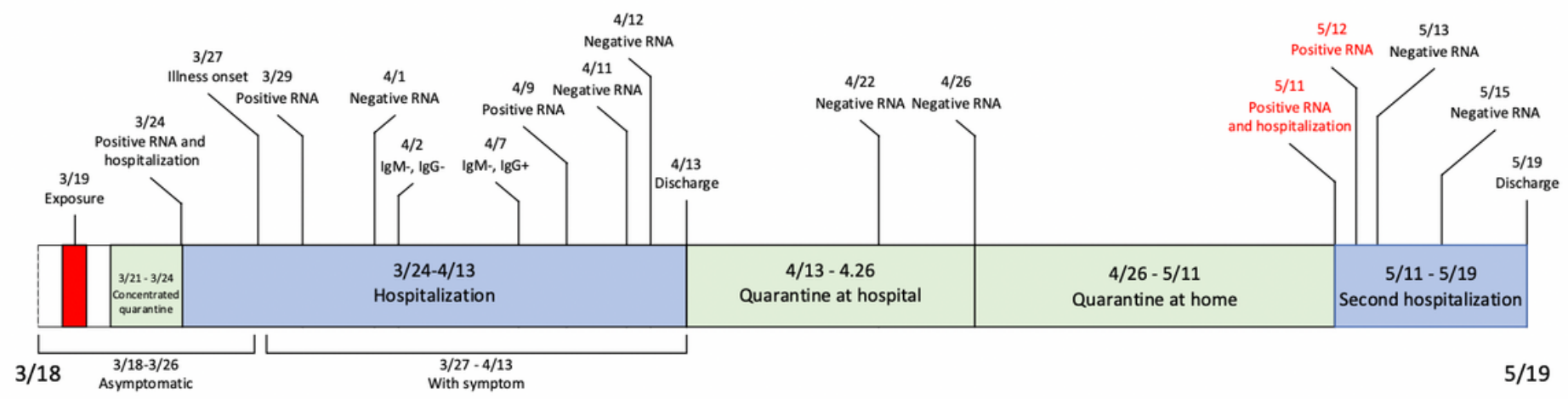

Figure 1

Timeline of recurrence of SARS-CoV-2 RNA from recovered patient with coronavirus disease 2019. 